\title{
Leadership contestability, monopolistic rents and growth
}

\author{
Roberto Piazza ${ }^{\dagger}$ \\ International Monetary Fund
}

This version: March 2011

\begin{abstract}
I construct an endogenous growth model where $R \& D$ is carried out at the industry level in a game of innovation between leaders and followers. Innovation costs for followers are assumed to increase with the technological lag from leaders. I obtain three results that contrast with standard Schumpeterian models, such as Aghion and Howitt (1992). First, leaders may innovate in equilibrium, in an attempt to force followers to drop out of the innovation game. Second, policies (such as patents) that allow for strong protections of monopolies can reduce the steady state growth rate of the economy. Third, multiple equilibria arise when monopolies' protection is large.
\end{abstract}

\section{INTRODUCTION}

Early formalizations of the Schumpeterian idea of creative destruction, such as Grossman and Helpman (1991), Aghion and Howitt (1992) provided two results. The first is that granting larger monopolistic rents to industry leaders should raise the growth rate of an economy, by spurring more innovation by followers. The second is that, because of the "replacement-effect", followers have more incentives to innovate than incumbent monopolists, who don't innovate in equilibrium.

On an empirical ground, these results have been challenged by the observation that monopolists do innovate and that there appears to be an inverted- $U$ relationship between monopolistic rents and innovation rates. This is essentially the point made by Aghion, Bloom, Blundell, Griffith, and Howitt (2005), who also present a theoretical model, based on the "escape competition" effect, that accounts for their empirical finding, in particular for the result that large monopolistic rents can decrease the overall innovation rate of an economy. Similar results are found in Aghion, Harris, Howitt and Vickers (2001). Building on this latter model, Acemoglu and Ufuk (2006) show that the patents

\footnotetext{
$\dagger$ I would like to thank Michele Boldrin for many stimulating discussions. The views expressed here are those of the author and do not necessarily reflect those of the International Monetary Fund. Any remaining errors are the authors' responsibility. Contact information: rpiazza@imf.org.
} 
policy that maximizes growth entails granting higher monopolistic rents to the monopolists that already have a large lead over their followers.

The studies cited above share a common feature: they assume that innovation costs for a follower doesn't depend on the distance from the leader ${ }^{1}$. The novelty of this paper is to construct an analytically simple endogenous growth model where this assumption is relaxed, and innovations costs are allowed to increase with the distance from the leader. From a theoretical perspective, the consequences turn out to be substantial, marking a departure from standard results in the literature on endogenous growth. Specifically I find that, even though current monopolistic profits of a leader don't depend on the distance from the follower (as it is, instead, in Aghion, Bloom, Blundell, Griffith, and Howitt [2005]), leaders may nonetheless innovate in equilibrium. Furthermore, when legal or technological barriers create an environment with strong protection of monopolies, multiple equilibria can arise. In high growth equilibria most of the industries are "neck-a-neck", i.e. the technological gap between leaders and the followers is usually small, and innovation is mostly done in equilibrium by followers, as in Aghion and Howitt (1992). Conversely, in low growth equilibria, followers do little innovation and have a large lag over the leaders, who undertake large but infrequent R\&D activities. Finally, I find that when monopolistic rents are too high, the only equilibrium is the low growth one. These results are consistent with the view (Boldrin and Levine [2008], Holmes and Schmitz [2010]) that sees lack of competition as a cause for low growth and innovation rates.

The assumption, crucial to this paper, that innovation costs for followers increase with the distance from the leader can be justified on various grounds, as the following examples attempt to argue. First of all, consider the role of patents. When technologies are patentable, a follower has only two options to win the leadership. The first is to build a new technology based on the leader's patent. This requires, of course, a license that the leader might not want to grant or would grant only at a very high price. The second is to avoid paying a licensing fee, but having to retrace all the missing steps that separate her from the state-of-the-art technology of the leader, an activity whose cost increases with the the distance from the leader. A second example is the case when, due to its great superiority, the leader's technology has become the "standard" product of an industry. Switching from a standard to a new product could be very costly for consumers (think for instance of the cost, in terms of software compatibility, that users have to incur to switch from a Windows-based to a Macintosh personal computer). Therefore, followers end up incurring large development and marketing costs to launch new products. A third example is the situation when innovation activities benefit from private knowledge about the state of an industry's technology. Even in the absence of formal patents, leaders may then retain some secret knowledge (the Coca-Cola formula) that put less experienced followers in disadvantaged position in the innovation race.

The role of state dependent innovation costs has been studied in the microeconomic literature in the context of "games of innovation" (Reinganum [1981], [1985]). In partial equilibrium settings, various papers (Harris and Vickers [1987], Budd, Harris and Vickers [1993] and Horner [2004]) have shown that, when the cost of leap-frogging the leader increases with the follower's lag, an important strategic reason for leaders to innovate emerges. By innovating, leaders are able to push the state (namely, the distance from the follower) of the industry to a point where progressively increasing

\footnotetext{
${ }^{1}$ A recent version of Acemoglu and Ufuk (2006) studies the possibility of slow step-by-step catch-up by the followers, which implies that the overall innovation effort that followers have to exert to become leaders is an increasing function of the followers' lag.
} 
costs eventually force followers to end any innovation activity. This is called "end-point" strategy for leaders. End-point strategies are at the core of our results, and are the cause of low-growth equilibria. The profitability of following an end-point strategy depends in principle on two factors. The first, is the size of monopolistic rents. The higher these rents, the more valuable it is for the leader to secure them by forcing the follower to drop out of the innovation race. The second is the presence of exogenous constraints that limit the duration of a leadership. For instance, frequent patent expirations reduce the cost disadvantage for laggards, neutralizing the usefulness of a leader's end-point strategy. Building on the above intuitions, this paper shows how the level of monopolistic rents and the exogenous (legal or technological) limits to the duration of a monopoly determine the equilibrium growth of the economy by affecting leaders' incentives to play end-point strategies.

The rest of the paper proceeds as follow. Section II and Section III introduce technologies and preferences of the economy. Section IV presents in details the game of innovation. Section V defines a stationary equilibrium and Section VI discusses the model's results. Section VII concludes.

\section{TECHNOLOGIES}

Time $t \geq 0$ flows continuously from zero to infinity. Three types of production activities occur at every instant $t$ : production of a final good, production of a continuum $i \in[0,1]$ of intermediate goods, and R\&D activities. The technology corresponding to each activity is presented below where, for simplicity, we omit time subscript from the variables.

\section{A. Final good production}

The final good production is represented by a constant returns to scale technology $F(\cdot)$ that transforms an intermediate input $X$, whose unit price in terms of the consumption numeraire is $P$, into an amount $Y=F(X)$ of consumption goods. We simply assume that

$$
Y=X
$$

The zero profit condition in the final good sector then requires

$$
P^{*}=1
$$

\section{B. Intermediate goods production}

The input $X$ is a Dixit-Stiglitz aggregator of intermediate goods quantities $x_{i}$ produced by a continuum of industries $i \in[0,1]$,

$$
X=\left[\int x^{\frac{1}{2}}(i) d i\right]^{2}
$$

At any time $t$ there are two firms in each industry, a leader and a follower. Leaders are more productive than followers, who do not produce the intermediate good. In each industry $i$, the leader produces the industry's intermediate good according to the linear technology,

$$
x(i)=a(i) l(i)
$$


where $l(i)$ is the amount of unskilled labor hired at the market wage $w$, and $a(i)$ is the leader's productivity. Call $\tilde{m}$ the mark-up applied by the leader over its (constant) marginal cost of production, namely

$$
\tilde{m}=\frac{p^{*}(i) a(i)}{w}
$$

where $p^{*}(i)$ is the price at which the leader decides to sell good $i$. Standard arguments imply that if the leader was to act as a pure monopolist it would choose $\tilde{m}=2$, where 2 is the elasticity of substitution among intermediate goods derived from the quadratic form of the aggregator $X$.

The extent of barriers to entry usually determine the size of the mark-up that a leader can impose to the market. Some of these barriers are due to market regulation policies, such as implementation of anti-trust laws or the degree of patents protection. Other barriers are more strictly technological, such as competitors' fixed costs of entry and production capacity limitations. All these elements determine the maximum (exogenous) mark-up $m$ that the leader can choose without being driven out of business (or fined). It is a simple exercise to show that, whenever the leader is allowed to choose any of the markups $\tilde{m} \leq m$, it will always choose

$$
\tilde{m}=m
$$

as long as $m \in[1,2]$, a restriction that from now on we assume to hold. The choice of a quadratic form for the aggregator $X$ allows us to greatly simplify the model, while at the same time retaining a sufficient level of generality in the study of our problem ${ }^{2}$.

At any instant, the price aggregator $P$ is given by

$$
P=\left[\int_{i \in[0,1]} p^{-1}(i) d i\right]^{-1}=\frac{m w}{A}
$$

where the aggregate productivity $A$ is simply,

$$
A=\int_{i \in[0,1]} a(i) d i
$$

Since $P^{*}=1$ the productivity adjusted salary $\hat{w}^{*}$ of unskilled workers is

$$
\hat{w}^{*} \equiv \frac{w}{A}=\frac{1}{m}
$$

As done above with the salary $w$, we will keep indicating with a symbol $\hat{\nu}$ the productivity-adjusted value of any variable $\nu$. Given a mark-up $m$, the inverse demand function for good $i$ implies that the optimal quantity $x^{*}(i)$ produced by the leader and its instantaneous profit $\pi^{*}(i)$ are

$$
\begin{gathered}
x^{*}(i)=\left[\frac{P^{*}}{p^{*}(i)}\right]^{2} X=\left(\frac{a(i)}{A}\right)^{2} X \\
\pi^{*}(i)=p^{*}(i) x^{*}(i)-\frac{w x^{*}(i)}{a(i)}=(m-1) a(i) \hat{w}^{*} \hat{X}
\end{gathered}
$$

\footnotetext{
${ }^{2}$ The quadratic form of $X$ allows us to obtain a simple linear for for the leader's profit function.
} 
The aggregate amount of unskilled labor $L$ used in the production of intermediate goods is

$$
L=\int_{i \in[0,1]} \frac{x^{*}(i)}{a(i)} d i=\hat{X}
$$

The variable $\hat{X}$ is then the aggregate demand of skilled labor by the intermediate goods sector. We can rewrite the leader's profits as

$$
\pi^{*}(i)=(m-1) a(i) \hat{w}^{*} \hat{X}
$$

Aggregate profits $\Pi$ of the intermediate goods sector are given by

$$
\Pi=\int_{i \in[0,1]} \pi(i) d i=(m-1) A \hat{w}^{*} \hat{X}=A \hat{\Pi}
$$

\section{Innovation technology}

Innovation is the activity through which firms improve their production technology. The state-of-the-art technology in any industry at any time is given by the leader's technology $a(i)$. Since we have assumed that only leaders produce and make profit, followers have incentives to innovate only if this allows them to become the new leader by increasing their productivity above the current state-of-the-art $a(i)$. We then assume that a successful innovation allows a firm to bring its productivity from a certain level $\tilde{a} \leq a(i)$ to a new level $\gamma a(i)$, where $\gamma>1$ is a parameter determining the size of a successful innovation. Clearly, a successful innovation done by a follower determines a change of leadership in the industry. Let us denote with a subscript " $f$ " any variable which refer to a follower, and with "l" a variable that refers to a leader.

To innovate and carry on R\&D activities, firms hire skilled labor $h$ at a wage $\omega(i) w$, with $\omega(i)$ the wage premium paid to skilled labor in industry $i$. A firm with current technological level $\tilde{a}$, employing an amount $h$ of skilled labor, in an industry with state-of-the art technology $a$, receives a successful innovation at a Poisson rate $\lambda$, where

$$
\lambda=\frac{A h}{\tilde{\xi}(\tilde{a}, a)}
$$

where $\tilde{\xi}(\cdot, \cdot)$ is a constant returns to scale cost function. This latter property, implies that we can write $\tilde{\xi}(\cdot, \cdot)$ as

$$
\tilde{\xi}(\tilde{a}, a)=a \xi(d)
$$

for some $\xi(\cdot)$ as a function of a (normalized) distance $d=\frac{\log a-\log \tilde{a}}{\log \gamma}$ between the firms's technology and the industry's state-of-the art technology. The R\&D production function can then be rewritten as

$$
\lambda=\frac{A h}{a \xi(d)}
$$

The unit $\operatorname{cost} \frac{\omega(i) w h}{\lambda}$ of $\lambda$ then depends on the efficiency $a(i) / A$ of an industry relative to the average efficiency of the economy. It is relatively more difficult to further improve technologies that are already cutting-hedge (those with a high ratio $a(i) / A$ ). 
We assume that,

$$
\xi^{\prime}>0, \quad \xi^{\prime \prime}>0
$$

Condition (6) is the crucial assumption of the model. Since innovation steps are of size $\gamma$, the distance $d$ of a firm from the industry's technological frontier is represented by a non-negative integer $d=0,1,2, \ldots$ In particular, at any time the leader is characterized by a distance $d_{l}=0$, and the follower by a distance $d_{f} \geq 1$. Convexity of the costs function $\xi(\cdot)$ suggests that, when the distance $d_{f}$ is small, the innovation cost $\xi\left(d_{f}\right)$ of the follower is similar to the cost of $\xi(0)$ of the leader. Instead, when $d_{f}$ grows large, the innovation cost goes to infinity and the follower must necessarily stop innovating. To simplify the problem, without loosing the main intuition, we assume that

$$
\begin{aligned}
& \xi(0)=\xi(1)=\xi>0 \\
& \xi(2) \geq \bar{\xi}=\frac{\gamma}{\rho}
\end{aligned}
$$

where $\rho>0$ is the household's rate of time preference presented in the next section. Conditions (7) imply that the innovation cost for the leader and the follower are the same and equal to (abusing a bit the notation) a constant $\xi>0$ as long as the follower is one step distant from the leader. If the distance of the follower is greater than 2 , its innovation cost rises above a level $\bar{\xi}$. Appendix 3 shows that in no circumstance a follower will choose to innovate when its cost is equal to or greater than $\bar{\xi}$, which happens when $d_{f} \geq 2$.

Because of (7), one might want to consider situations where distances $d_{f} \geq 2$ do not represent absorbing states (more precisely, an ergodic set) for the follower's distance. To do so, we assume that at a given rate $\tau>0$ the distance $d_{f}$ of a follower from the leader is exogenously reduced back to 1 . The arrival rate $\tau$ can be interpreted, for instance, as the frequency at which the leader's patent expires (as in Acemoglu and Ufuk [2006]) or as the frequency of knowledge spill-overs from the leader to the follower. Knowledge spill-overs allow followers to move from state $d_{f} \geq 2$ where costs are too high to carry over R\&D to the state $d_{f}=1$ where the follower is on par with the leader in its ability to innovate. For this reason, we call $\tau$ the degree of leadership contestability and we refer to states where $d_{f}=1$ as states where the leadership is contestable, and to states $d_{f} \geq 2$ as states where the leadership is non contestable.

\section{PREFERENCES}

The problem of the representative household is to choose a flow of consumption $\left(C_{t}\right)_{t \geq 0}$, the evolution of debt $\left(B_{t}\right)_{t \geq 0}$ and the allocation of her time endowment. Specifically, the household has one unit of free time that can be used to supply unskilled labor $L$ to the intermediate goods sector or to generate skilled labor $H(i)$ for $\mathrm{R} \& \mathrm{D}$ activities in $i$. Given a time evolution of the interest rate $\left(r_{t}\right)_{t \geq 0}$, the wage of unskilled labor $\left(w_{t}\right)_{t \geq 0}$, the skill premiums $\left(\omega_{t}(i)\right)_{t \geq 0}$ in each industry $i$, profits $\left(\Pi_{t}\right)_{t \geq 0}$ gained from firms' ownership, and exogenous constraints $\bar{H}_{t}(i)$ and $\bar{B}$, the household solves

$$
\begin{gathered}
\max _{C_{t}, L_{t}, H_{t}(i)} \int_{0}^{\infty} \log C_{t} d t \\
\text { s.t. } \quad C_{t}+\dot{B}_{t}=r_{t} B_{t}+w_{t} L_{t}+w_{t} \int \omega_{t}(i) H_{t}(i) d i+\Pi_{t}
\end{gathered}
$$




$$
\begin{gathered}
L_{t}+\int H_{t}(i) d i=1 \\
B_{t} \leq \bar{B} \\
H_{t}(i) \leq \bar{H}_{t}(i)
\end{gathered}
$$

The arbitrary constant $\bar{B}>0$ is used to avoid Ponzi games and is never binding in equilibrium. We allow for debt $B_{t}$ only to obtain the equalibrium value for the interest rate $r_{t}$. Each unit of free time can be used by the houshold to produce one unit of skilled labor. Condition (11) sets exogenous bounds on the amount of skilled labor that can be supplied to each industry. These bounds are an extreme form of decreasing marginal returns in industry-specific skilled labor production. The bounds are introduced to allow supplies $H(i)$ to be finite even when the wage premiua $\omega(i)$ are strictly greater than one. Condition (11) might then be binding in equilibrium, but by appropriately taking limits we can make these bounds arbitrarily large. To do this, we proceed as follows. First we assume that,

$$
\bar{H}_{t}(i)=\frac{\xi a_{t}(i)}{A_{t}} \bar{\Lambda}
$$

for some $\bar{\Lambda}>0$, and then we take limits for $\bar{\Lambda} \rightarrow \infty$. Comparing (12) with (5) we see that $\bar{\Lambda}$ is the maximum arrival rate of innovation which is feasible at any time in any industry.

\section{THE INNOVATION PROBLEM FOR $\gamma$ SMALL}

When an industry is in the contestable state $d_{f}=1$, the follower's incentive to innovate is to gain the rents from becoming the new leader. The innovation incentives for the leader are instead two. The first is to increase profits by innovating its technology. The second is to push the industry (the "end-point" strategy) to the uncontestable state $d_{f}=2$, where the follower stops being a threat to the leadership. The standard Arrow effect implies that one should expect the first to be a weak incentive to innovation for the leader. To focus more the attention on "end-point" strategies, which are the focus of the paper, we assume that

$$
\xi \geq \frac{\gamma-1}{\rho}
$$

Appendix 3 shows that under condition (13) the leader has never incentives to conduct R\&D at non contestable states $d \geq 2$. Assumption (13) is not necessary for the main results of the model, but is shortens the exposition ${ }^{3}$.

Within each industry, leaders and followers play a game of innovation, and expectations about each other's future strategies determine current innovation efforts. We employ a simple equilibrium concept for the game. We postulate (and later verify) that, on a balance growth path, there exists a

\footnotetext{
${ }^{3}$ The full version of the model where (13) may not hold is a simple extension of the model developed here, and is available from the author upon request
} 
Markov perfect equilibrium where the state space of the innovation game is given by the distance ${ }^{4}$ $d_{f}=1,2$ between the follower and the leader.

Specifically, on a balanced growth, $r^{*}, \hat{w}^{*}, \hat{X}^{*}$ are constant. The wage premium $\omega_{1}^{*}$ and $\omega_{2}^{*}$ for, respectively, skilled labor in a contestable and non contestable industry are also constant. Recall that the profit function and the innovation costs are defined by (3) and (5). Hence, it is straightforward to see that the value $V_{t}$ of a firm at time $t$ in an industry $i$ where the state-of-the-art technology is $a_{t}(i)$ and the distance of the follower $f$ from the leader $l$ is $d_{f}=1,2$ is given by

$$
V_{t}=a_{t}(i) \hat{V}_{d_{f}}^{j}
$$

where $\hat{V}_{d_{f}}^{j}$ are four (normalized by $a$ ) values defined as follows. Leaders and followers don't innovate at the non contestable state $d_{f}=2$, so their normalized values are given by, respectively,

$$
\begin{aligned}
r^{*} \hat{V}_{2}^{l} & =(m-1) \hat{w}^{*} \hat{X}^{*}+\tau\left(\hat{V}_{1}^{l}-\hat{V}_{2}^{l}\right) \\
r^{*} \hat{V}_{2}^{f} & =\tau\left(\hat{V}_{1}^{f}-\hat{V}_{2}^{f}\right)
\end{aligned}
$$

At the contestable state $d_{f}=1$, the leader takes as given the strategy $\lambda_{f}^{*}$ for the arrival rate of the follower and chooses an arrival rate $\lambda_{l}$ for its innovation,

$$
r^{*} \hat{V}_{1}^{l}=\max _{\lambda_{l} \geq 0}(m-1) \hat{w}^{*} \hat{X}^{*}-\xi \omega_{1}^{*} \hat{w}^{*} \lambda_{l}+\lambda_{l}\left(\gamma \hat{V}_{2}^{l}-\hat{V}_{1}^{l}\right)+\lambda_{f}^{*}\left(\gamma \hat{V}_{1}^{f}-\hat{V}_{1}^{l}\right)
$$

Similarly, at $d_{f}=1$ the follower takes as given the arrival rate $\lambda_{1}^{*}$ and chooses its arrival rate $\lambda_{f}$ to solve

$$
r^{*} \hat{V}_{1}^{f}=\max _{\lambda_{f} \geq 0}-\xi \omega_{1}^{*} \hat{w}^{*} \lambda_{f}+\lambda_{f}\left(\gamma \hat{V}_{1}^{l}-\hat{V}_{1}^{f}\right)+\lambda_{l}^{*}\left(\gamma \hat{V}_{2}^{f}-\hat{V}_{1}^{f}\right)
$$

\section{EQUILIBRIUM}

Industries in the contestable state $d_{f}=1$ experience growth in their state-of-the art technologies at the aggregate rate $\Lambda^{*}$,

$$
\Lambda^{*}=\lambda_{f}^{*}+\lambda_{l}^{*}
$$

There is no productivity growth, instead, in an industry's productivity in the non contestable state $d_{f}=2$. Since arrivals of innovations are independent across industries, it follows that the balanced growth rate $g^{*}$ of aggregate productivity (and thus of the economy) is given by,

$$
g^{*}=\frac{\dot{A}}{A}=(\gamma-1) \Lambda^{*} \alpha^{*}
$$

where $\alpha^{*}$ is defined as the stationary share of aggregate productivity allocated in equilibrium to industries in the contestable state. Industries exit the contestable state (with a net improvement $\gamma-1$ in their productivity) to enter the non contestable state at the equilibrium rate $\lambda_{l}^{*}$, and exit the non contestable state (leaving their productivity unchanged) to enter the contestable state at the

\footnotetext{
${ }^{4}$ Because of our assumptions, at states $d_{f} \geq 2$ neither the leader nor the follower innovate. Also, at a rate $\tau>0$ states with $d_{f} \geq 2$ transition to the state $d_{f}=1$. It follows that states $d_{f} \geq 3$ are never reached in the long run. Therefore, for expositional simplicity, we state the innovation problem only for distances $d_{f}=1,2$.
} 
exogenous rate $\tau$. While remaining in the contestable state, an industry's productivity improves (by a net fraction $\gamma-1$ ) at the rate $\lambda_{f}^{*}$. Given $\lambda_{1}^{*}, \lambda_{2}^{*}$ and $\tau$, Appendix 2 shows that the stationary value $\alpha^{*}$ must solve the quadratic equation

$$
(\gamma-1) \Lambda^{*} \alpha^{* 2}-\left(\gamma \lambda_{f}^{*}-\Lambda^{*}-\tau\right) \alpha^{*}-\tau=0
$$

We will later show that, once we substitute for the equilibrium condition for $\lambda_{f}^{*}$, condition (34) reduces to an equation of degree one where $\gamma$ does not appear. For this reason ${ }^{5}$ we will can to interpret $\alpha^{*}$ simply as the stationary fraction of industries that on the balanced growth are in the contestable state.

The household's choice to supply skilled labor can be restated in an aggregate form. Call $H_{1}^{*}$ and $H_{2}^{*}$, respectively, the stationary aggregate skilled labor supplied to industries offering a wage premium $\omega_{1}^{*}$ and $\omega_{2}^{*}$. The quantity $\omega_{1}^{*} H_{1}^{*}+\omega_{2}^{*} H_{2}^{*}$ can then be substituted for the integral in (8). Aggregating constraints (11) and (12) across industries $i$ gives,

$$
\begin{aligned}
& H_{1}^{*} \leq \xi \bar{\Lambda} \alpha^{*} \\
& H_{2}^{*} \leq \xi \bar{\Lambda}\left(1-\alpha^{*}\right)
\end{aligned}
$$

We can then drop (11)-(12) from the household's problem and substitute ${ }^{6}$ it with (19).

We are now ready to define a stationary equilibrium,

Definition 1. A stationary equilibrium is given by a growth rate $g^{*}$ for the aggregate productivity, an initial condition $B_{0}$, wages $\hat{w}$, profits $\hat{\Pi}^{*}$, output $\hat{Y}^{*}$, consumption $\hat{C}^{*}$, debt $\hat{B}^{*}$, wage premia $\left\{\omega_{d}^{*}\right\}_{d=1,2}$, a supply $L^{*}$ of unskilled labor, supplies $\left\{H_{d}^{*}\right\}_{d=1,2}$ of skilled labor, a demand $\hat{X}^{*}$ of unskilled labor, arrival rates $\left\{\lambda_{j}^{*}\right\}_{j=l, f}$ and values $\left\{\hat{V}_{d}^{j}\right\}_{d=1,2}^{j=l, f}$ such that,

1. Given $B_{0}, r^{*}, \hat{w}^{*},\left\{\omega_{d}^{*}\right\}, \hat{\Pi}^{*}, \alpha^{*}$ and the growth rate $g^{*}$ for the productivity, then labor supplies $L^{*}$ and $\left\{H_{d}^{*}\right\}_{d=1,2}$, and productivity-adjusted consumption $\hat{C}^{*}$ and debt $\hat{B}^{*}$ solve the household's problem.

2. Policies $\left\{\lambda_{j}^{*}\right\}$ and values $\left\{\hat{V}_{d}^{j}\right\}$ are an equilibrium of the innovation game.

3. $\alpha^{*}$ solves (34) and $g^{*}$ is given by (35).

4. Markets clear:

i) $\hat{B}^{*}=0$

ii) $\hat{C}^{*}=\hat{Y}^{*}$

iii) $\hat{X}^{*}=L^{*}$

iv) $H_{1}^{*}=\xi \Lambda^{*} \alpha^{*}, H_{2}^{*}=0$

\footnotetext{
${ }^{5}$ If $\alpha^{*}$ is independent on $\gamma$ then, to calculate its equilibrium level, we can simply assume $\gamma=1$. In this case, the entry end exit rates of productivities mentioned above are equivalent to entry and exit rates of industries.

${ }^{6}$ The reason why constraint (19) is equivalent to (11)-(12) is presented in Section A.
} 


\section{A. Equilibrium conditions for the households}

The household faces the inter-temporal problem of choosing the dynamics of consumption and the static problem of allocating the unit measure of labor. The standard Euler equation in the case of logarithmic utility gives

$$
\frac{\dot{C}}{C}=r^{*}-\rho
$$

In a balanced growth, consumption grows at the constant rate $g^{*}$ an then

$$
r^{*}=\rho+g^{*}
$$

An arbitrage condition implies that the household is also indifferent between supplying skilled and unskilled labor as long as $\omega_{1}=\omega_{2}^{*}=1$. However, if for some $d_{f}=1,2$ we have industry $\omega_{d_{f}}(i)>1$, then (19) binds,

$$
\begin{array}{ll}
H_{1}^{*}=\xi \bar{\Lambda} \alpha^{*} & \text { if } \omega_{1}^{*}>1 \\
H_{2}^{*}=\xi \bar{\Lambda}\left(1-\alpha^{*}\right) & \text { if } \omega_{2}^{*}>1
\end{array}
$$

In equilibrium we must have $H_{2}^{*}=0$ so, without loss of generality, we set $\omega_{2}^{*}=1$. The market clearing condition for $H_{1}^{*}$ implies

$$
H_{1}^{*}-\xi \bar{\Lambda} \alpha^{*}=\xi \alpha^{*}\left(\Lambda^{*}-\bar{\Lambda}\right)
$$

In equilibrium, condition (19) is then equivalent to,

$$
\Lambda^{*} \leq \bar{\Lambda}
$$

As long as (22) holds, then also (11)-(12) hold for any industry $i$, explaining why conditions (11)-(12) are equivalent to (19).

\section{B. Equilibrium with no monopolistic innovation: $\lambda_{l}^{*}=0$.}

In this section we present the conditions that must hold in an equilibrium where only followers innovate, so that $\alpha^{*}=1$ and

$$
g^{*}=(\gamma-1) \lambda_{f}^{*}
$$

Because of constant returns in the innovation technology, a solution for the innovation problem of the follower at $d_{f}=1$ exists only if

$$
\xi \omega_{1}^{*} \hat{w} \leq \gamma \hat{V}_{1}^{l}-\hat{V}_{1}^{f}
$$

implying that

$$
\hat{V}_{1}^{f}=0
$$

When leaders never innovate their value at $d_{f}=1$ is given by

$$
\hat{V}_{1}^{l}=\frac{(m-1) \hat{w} \hat{X}}{r^{*}+\lambda_{f}^{*}}
$$


Therefore, followers innovate if and only if

$$
\xi \omega_{1}^{*}=\frac{\gamma(m-1) \hat{X}^{*}}{r^{*}+\lambda_{f}^{*}}
$$

Let us for a moment assume that (22) is not binding, so that $\omega_{1}^{*}=1$ and labor market clearing implies that $\hat{X}^{*}=1-H_{1}^{*}=1-\xi \lambda_{f}^{*}$. Substituting this condition and (20) into (23) we conclude that in a equilibrium where only followers innovate the arrival rate $\lambda_{f}^{*}>0$ is given by

$$
\lambda_{f}^{*}=\frac{\gamma(m-1)-\rho \xi}{\xi \gamma m}
$$

If for some $m$ equation (24) gives a negative number there is no equilibrium where followers carry on strictly positive R\&D, since rents $m$ from becoming a leader don't compensate for the innovation costs $^{7}$. It is also straightforward to see that we can always choose a value $\bar{\Lambda}$ sufficiently large that (22) is not binding for any $m \in[1,2]$.

As a last step, we need now to verify that when (24) holds then leaders have indeed no incentive to innovate. A leader does not innovate at $d_{f}=1$ if and only if

$$
\xi \omega_{1}^{*} \hat{w} \xi \geq \gamma \hat{V}_{2}^{l}-\hat{V}_{1}^{l}
$$

Writing $\hat{V}_{2}^{l}$ as a function of $\hat{V}_{1}^{l}$ and then substituting for the value of $\xi \omega_{1}^{*}$ in (23) we obtain

$$
\gamma \lambda_{f}^{*} \leq r^{*}+\tau
$$

Since the growth rate satisfies $g^{*}=(\gamma-1) \lambda_{f}^{*}$, condition (25) implies that the maximum arrival rate that followers could reach in equilibrium before triggering innovation by the leader is given by

$$
\lambda_{f}^{*} \leq \rho+\tau
$$

Using the equilibrium value (24) for $\lambda_{f}^{*}$, we can also restate (25) in terms of rents $m$ by saying that an equilibrium where leaders don't innovate exists as long as

$$
\begin{aligned}
m & \leq \bar{M} \\
\bar{M} & \equiv \frac{\gamma+\rho \xi}{\gamma(1-\rho \xi-\tau \xi)}
\end{aligned}
$$

\section{Equilibria with monopolistic innovation: $\lambda_{l}^{*}>0$.}

Equation (25) provides a clear picture for why leaders may want to innovate at the contestable state. For a given interest rate $r^{*}$, pushing the industry to the non contestable state $d_{f}=2$ is more valuable to the leader when the threat $\lambda_{f}^{*}$ of a takeover by the follower is large. It is also more valuable when the the degree of contestability $\tau$ is small, since in these cases the state $d_{f}=2$ becomes very "safe". In an equilibrium where both the leader and the follower innovate, equation (25) must hold with equality, giving

$$
\gamma \lambda_{f}^{*}=\rho+\tau+g^{*}
$$

\footnotetext{
${ }^{7}$ In this case the equilibrium is with $\lambda_{f}^{*}=g^{*}=0$.
} 
Substituting (35) into (28) and then inserting the resulting expression for $\lambda_{f}^{*}$ into (34) provides a unique solution for $\alpha^{*}$,

$$
\alpha^{*}\left(\Lambda^{*}\right)=\frac{\tau}{\Lambda^{*}-\rho}
$$

Since condition (23) for innovation by the followers must also hold, we obtain

$$
\xi \omega_{1}^{*}=\frac{\gamma(m-1)\left(1-\xi H^{*}\right)}{r^{*}+\lambda_{f}^{*}}=\frac{\gamma^{2}(m-1)\left[1-\xi \alpha^{*}\left(\Lambda^{*}\right) \Lambda^{*}\right]}{(\gamma+1) \rho+\tau+\left(\gamma^{2}-1\right) \alpha^{*}\left(\Lambda^{*}\right) \Lambda^{*}}
$$

Given that $\omega^{*} \geq 1$ and since the right hand-side of the equation above is decreasing in $\Lambda^{*} \leq \bar{\Lambda}$ we conclude that a necessary and sufficient condition of the existence of an equilibrium where leaders innovate is

$$
\xi \leq \frac{\gamma^{2}(m-1)\left[1-\xi \alpha^{*}(\bar{\Lambda}) \bar{\Lambda}\right]}{(\gamma+1) \rho+\tau+\left(\gamma^{2}-1\right) \alpha^{*}(\bar{\Lambda}) \bar{\Lambda}} \stackrel{\bar{\Lambda} \rightarrow \infty}{\longrightarrow} \frac{\gamma^{2}(m-1)(1-\xi \tau)}{(\gamma+1) \rho+\gamma^{2} \tau}
$$

Again, we can restate the condition above in terms of rents $m$ and say that an equilibrium where leaders innovate exists if and only if

$$
\begin{aligned}
m & \geq \frac{M}{[(\gamma+1) \rho+\tau] \xi+\gamma^{2}-\xi \alpha^{*}(\bar{\Lambda}) \bar{\Lambda}} \\
\underline{M} & \equiv \frac{\bar{\Lambda} \rightarrow \infty}{\longrightarrow} \frac{(\gamma+1) \rho \xi+\gamma^{2}}{1-\tau \xi} \frac{(\bar{\Lambda}) \bar{\Lambda}}{\stackrel{-1}{\longrightarrow}}
\end{aligned}
$$

For any value of $\bar{\Lambda}$, it is easy to show ${ }^{8}$ that $\underline{M}<\bar{M}$.

\section{RESUlts}

The next proposition provides the characterization of the equilibria of the model.

Proposition 1. An equilibrium always exists. For values $\bar{M}$ and $\underline{M}$ defined by (27) and (30) we have that

i) An equilibrium with $\lambda_{l}^{*}>0$ and $\bar{\Lambda}$ not binding always exists if $m \in(\underline{M}, \bar{M})$.

ii) An equilibrium with $\lambda_{l}^{*}>0$ and $\bar{\Lambda}$ binding always exists if $m \geq \underline{M}$. Moreover, if $m>\bar{M}$ it is also the unique equilibrium of the model.

iii) If $m \in[1, \bar{M}]$ there exists one and only one equilibrium with $\lambda_{l}=0$.

iv) When, for $m \in[\underline{M}, \bar{M}]$, equilibria are multiple, the growth rate $g^{*}$ of the equilibrium in iii) is strictly bigger than growth in i), which is strictly bigger than growth in ii).

Proof. See Appendix 1.

Q.E.D.

${ }^{8}$ Choose $\bar{\Lambda}^{*}=\rho+\tau$ and find that $\bar{M}<\underline{M}$. Therefore, for any $\bar{\Lambda}>\rho+\tau$ we must have $\bar{M}>\underline{M}$. 
An interesting corollary to Proposition 1 is the following,

Corollary 1. i) Given rents $m$, the maximal equilibrium growth for the economy is reached when the degree of leadership contestability $\tau$ is sufficiently large,

$$
\tau \geq \bar{\tau}=\frac{2(\gamma+1) \rho \xi-\gamma^{2}}{2 \xi}
$$

In this case the equilibrium is unique and $\lambda_{l}^{*}=0$.

ii) Given $\tau$, the growth rate attained a the (unique) equilibrium when $m>\bar{M}$ is smaller than the growth rate attained at any equilibrium with $m \in[\underline{M}, \bar{M}]$.

Proof. For part $i$ ) it is easy to see that for $\tau \geq \bar{\tau}$ we have $\underline{M}>2$. Figure 1 makes then clear that the equilibrium in this case is unique, and is the high-growth equilibrium where only followers innovate. Part $i$ ) is also a straightforward conclusion that can be drawn from Figure 1.

Q.E.D.

Figure 1 gives a graphical representation of the results in Proposition 1 and in Corollary 1. We obtain four main conclusions. First, in the presence of state-dependent innovation costs there might exist equilibria where both leaders and followers innovate. Second, leaders innovate only to push their industry to non contestable states ("end-point" strategy) where innovation stops, and thus equilibria where leaders innovate are characterized by low growth $g^{*}$. Third, increasing rents $m$ beyond a certain threshold $\bar{M}$, causes a reduction in the equilibrium growth that an economy can attain. Fourth, by making leadership's contestability sufficiently large $(\tau>\bar{\tau})$, the multiplicity of equilibria disappears, and only the high growth equilibrium, where leaders don't innovate, survives.

We can gain intuition for the conclusions above by giving a more detailed look at Figure 1 . When rents $m$ are low there is no innovation since it is not worthy for followers to spend resources to gain a leadership that pays little. Since leaders are not threatened, they don't innovate either. For rents in the range $[1+\rho \xi / \gamma, \underline{M}]$ followers innovate, but their innovation rate is still too low to be a substantial threat for leaders.

When $m \geq \underline{M}$ multiple equilibria appear. The equilibrium with the highest growth is the one where only followers innovate and industries are always in the contestable state $\left(\alpha^{*}=1\right)$. The large arrival rate of followers now represents a substantial threat for the leaders. However, this effect is counterbalanced by the fact that, in this high-growth equilibrium, the interest rate $r^{*}$ at which profits are discounted is large. Moreover, the amount $L^{*}=1-H_{1}^{*}$ of unskilled labor available to the intermediate goods sector is low, thus reducing output and profits of leaders. Overall, even in face of moderately high innovation rates $\lambda_{f}^{*}$ by followers, the value of securing the leadership at the non contestable state is still relatively small, and thus leaders choose not to innovate.

The equilibrium with medium-level growth is characterized by some innovation done by the leaders, but this innovation effort is not so large to make $\bar{\Lambda}$ bind. The moderate innovation rate by the leaders positions the share $\alpha^{*}$ of industries in the contestable state at an intermediate level $\alpha^{*} \in(\tau /(\bar{\Lambda}-\rho), 1)$. Since, at every instant, some industries are in the non contestable state, where no innovation takes place, the growth rate of the economy is lower than in the high-growth equilibrium. Consequently, the interest rate $r^{*}$ and the supply of skilled workers $H^{*}$, are also smaller. This increases the value of a leadership above that in the high-growth equilibrium, explaining why leaders innovate and make some effort to reach the non contestable state.

In the low equilibrium, the economy perpetuates a situation where only a small fraction 


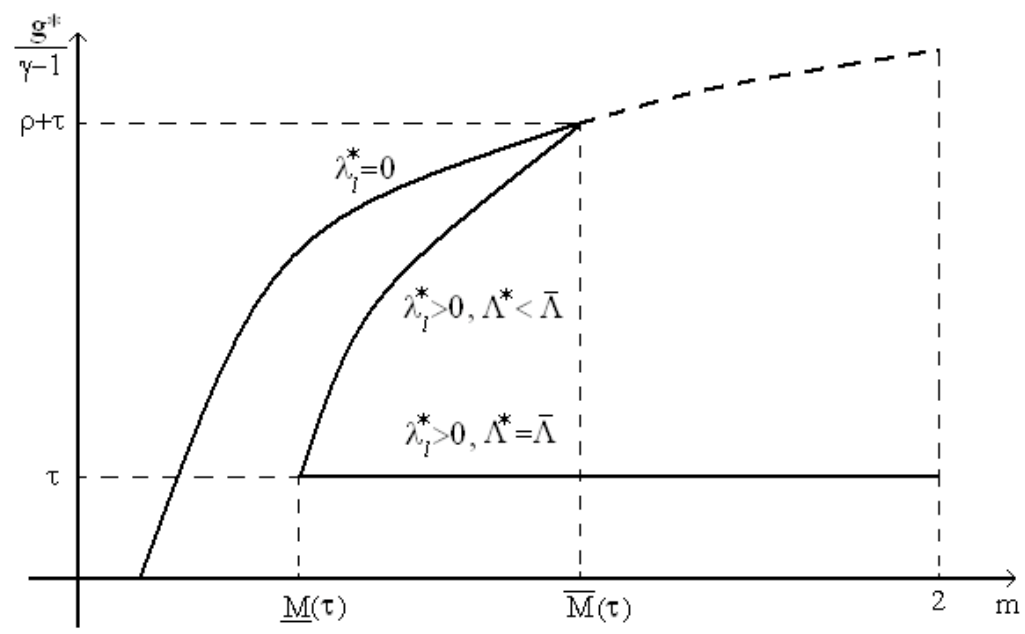

Figure 1. Loci of (normalized) equilibrium growth rates $\frac{g^{*}}{\gamma-1}=\Lambda^{*} \alpha^{*}$ assuming $\bar{\Lambda} \rightarrow \infty$ and $\bar{M}<2$. For $m \in(\underline{M}, \bar{M})$ the high-growth equilibrium corresponds to $\lambda_{l}^{*}=0$, the medium-growth equilibrium to $\lambda_{l}^{*}>0$ and $\Lambda^{*}<\bar{\Lambda}$, the low-growth equilibrium to $\lambda_{l}^{*}>0$ and $\Lambda^{*}=\bar{\Lambda}$.

$\alpha^{*}=\tau /(\bar{\Lambda}-\rho)$ of the industries are in the contestable state. For $\bar{\Lambda}$ going to infinity, the fraction $\alpha^{*}$ goes to zero and growth $g^{*}=(\gamma-1) \bar{\Lambda} \alpha^{*}(\bar{\Lambda})$ tends to a multiple $g^{*}=(\gamma-1) \tau$ of the degree of leadership contestability $\tau$. This is an equilibrium because when growth is low $r^{*}$ and $H^{*}$ are low too, substantially increasing the value of the leadership. Leaders have then a large incentive to secure their profits by reaching the non contestable state. The innovation rate of a leader is so large that $\bar{\Lambda}$ binds. In the limit, while the innovation rate of a follower is $\lambda_{f}^{*}=\tau+\frac{\rho}{\gamma}$, the innovation rate $\lambda_{l}^{*}=\bar{\Lambda}-\lambda_{f}^{*}$ of a leader tends to infinity. This implies that, while leaders are forced back to the contestable state at a rate $\tau$, their innovation effort $\lambda_{l}^{*}$ is so large that the industry immediately returns to the non contestable state. The low-growth equilibrium is a situation when massive innovation is done by leaders in the rare occurrence that their industry is in the contestable state.

Finally, for $m>\bar{M}$ only the low-growth equilibrium exists. If this wasn't the case, rents $m$ and innovation rates $\lambda_{f}^{*}$ would be so high $\left(\lambda_{f}^{*}>\rho+\tau\right)$ that, despite possible high values for $r^{*}$ and $H^{*}$, they would trigger a massive incentive for leaders to escape the contestable state. This would be consistent only with an equilibrium with a low $\alpha^{*}$.

Corollary 1 tackles some issues that might be relevant for policy purposes. If the goal of a regulator is to maximize growth, then the best policy is to maximize the degree of leadership contestability $\tau$, for instance by forcing leaders to freely allow followers to use their patents for the purpose of building innovation upon the leader's product. When $\tau$ is large enough there is no reason for leaders to innovate, since even in the event where they reached the non contestable state, they would be almost immediately brought back to the contestable state. With $\tau$ large the high-growth equilibrium is the only equilibrium of the economy (the solid dashed line in Figure 1). Even partial increases in $\tau$ might have a beneficial effect on growth (notice, in particular, that $\underline{M}$ and $\bar{M}$ are increasing in $\tau$ ).

When $\tau$ is large, the model becomes equivalent under many respects to Aghion and Howitt (1992) and in this case (and only in this case!) an increase in the level of rents $m$ is guaranteed to improve the growth rate of the economy. Instead, if the degree of contestability is small, an increase in rents 
(due, for instance, to an increase in patents protection) might cause the equilibrium growth rate to fall. In fact, if $m$ is increased beyond $\bar{M}$, the only equilibrium of the economy is the low-growth equilibrium ${ }^{9}$. This point can be summarized by saying that high rents and low degree of contestability are as harmful for growth as the absence of seizable rents.

\section{Conclusions}

We have constructed an endogenous growth model where $R \& D$ in carried out at the industry level in a game of innovation between industries' leaders and followers. The novelty of our approach is to relaxed the assumption, usually made, that innovation costs for followers are state-independent. Instead, we allow for innovation costs to increase with the follower's distance (the state of an industry) from the state-of-the-art technology of the leader. We use two exogenous parameters to represent institutional or technological factors that influence equilibrium strategies for the game of innovation. The first is the size of rents appropriated by an industry's leader. The second is the frequency, that we call degree of leadership contestability, at which exogenous knowledge spill-overs reduce the distance between leaders and followers. We focus on the balanced growth paths of the model and we obtain results that are new to the growth literature. First of all, even though monopolistic profits are independent on the industry's state, leaders nonetheless might innovate in equilibrium. Leaders' innovation activity is aimed at pushing the industry's state to points where innovation costs are so large for followers that they stop innovating, ending the threat to the leadership. This is a leader's "endpoint" strategy.

Typically, when the degree of leadership contestability is low, multiple equilibria can arise. The equilibrium with the highest growth rate is always the equilibrium where leaders don't innovate. This is the typical equilibrium, for instance, in Aghion and Howitt (1992) or Grossman and Stiglitz (1991). Equilibria with strictly positive innovation by leaders feature lower growth because, even though leaders" effort contribute to raise the overall R\&D of their industry (the "intensive margin" of innovation) it also increases the frequency (the "extensive margin") at which industries end up in states where innovation does not occur, since the distance between the leader and the follower is large.

An exogenous increase in the size of monopolistic rents is not guaranteed to stimulate more aggregate innovation. Indeed, when the degree of market contestability is low and monopolistic rents are raised beyond a certain threshold, the high-growth equilibrium disappears and the only stationary equilibrium is the one with low growth. This equilibrium is characterized by moderate innovation by followers and and large (but unfrequent) innovation by leaders.

Finally, when the degree of leadership contestability is large, the multiplicity of equilibria disappears, and only the traditional high-growth equilibrium, with no innovation by leaders,

\footnotetext{
${ }^{9}$ Interestingly, an increase in rents $m$ does not change the growth level in the low-growth equilibrium. This is because the "intensive margin" of innovation $\Lambda^{*}$ has already reached its upper bound $\bar{\Lambda}$, while the "extensive margin " $\alpha^{*}(\Lambda)$ is fixed and pinned down by $\bar{\Lambda}$. Increases in $m$ have then the only effect to increase profits $\hat{\pi}^{*}$, by the decreasing wages $\hat{w}^{*}$, and increasing wage premia $\omega_{1}^{*}$ in industries where $\bar{\Lambda}$ binds.

When (13) does not hold, leaders may choose to innovate at states $d_{f} \geq 2$. The only change to Figure 1 would be that growth in the low-growth equilibrium rises with $m$ (the high growth and the medium-growth are unaffected), but still remains lower than the medium and high growth equilibria.
} 
survives. In this case (and only in this case) an increase in the size of monopolistic rents unambiguously rises aggregate growth. 


\section{REFERENCES}

Acemoglu D. and Ufuk A. (2006), State Dependent Intellectual Property Rights Policy, NBER Working Paper No. 12775.

Aghion P., Bloom N., Blundell R., Griffith R., Howitt P. (2005), Competition and Innovation: An Inverted-U Relationship, The Quarterly Journal of Economics 120, 701-728.

Aghion P, Harris C., Howitt P., Vickers J. (2001), Competition, Imitation and Growth with Step-by-Step Innovation, Review of Economic Studies 68, 467-492.

Aghion and Howitt (1992), A Model of Growth Through Creative Destruction,? Econometrica 110, 323-351.

Boldrin M., Levine D.K. (2008), Against Intellectual Monopoly, Cambridge University Press.

Budd, Harris and Vickers (1993), A Model of the Evolution of Duopoly: Does the Asymmetry Between Firms Tend to Increase or Decrease?, ? Review of Economic Studies 60, 540-573.

Grossman G.M., Helpman E. (1991), Quality ladders in the theory of growth, Review of Economic Studies 58, 43-61.

Harris and Vickers (1987), Racing with Uncertainty, Review of Economic Studies 54, 1-21.

Holmes T.J., Schmitz J.A. (2010), Competition and Productivity: A Review of Evidence, Federal Reserve Bank of Minneapolis, Research Department Staff Report 439.

Horner J. (2004), A perpetual race to stay ahead, Review of Economic Studies 71, 1065-1088.

Reinganum, J. (1981), Dynamic Games of Innovation, Journal of Economic Theory 25, 21-24.

Reinganum, J. (1985), Innovation and Industry Evolution, Quarterly Journal of Economics 100, 81-100. 


\section{Appendix 1. Proof of Proposition 1.}

Point $i$ ) is proven as follows. We know that, by construction of $\underline{M}$, if $m=\underline{M}$ there exists an equilibrium where $\Lambda^{*}=\bar{\Lambda}$ and $\omega^{*}(a, 1, A)=\xi$. Since the right-hand side of (29) is strictly increasing in both $\Lambda^{*}$ and $m$, there is a one-to-one (strictly decreasing) correspondence between values $m>\underline{M}$ and values of $\Lambda^{*}<\bar{\Lambda}$ so that (29) is satisfied with $\omega^{*}(a, 1, A)=\xi$. This correspondence defines equilibria with $\lambda_{l}^{*}>0$ and $\Lambda^{*}<\bar{\Lambda}$. In particular, as $m$ increases, $\Lambda^{*}$ decreases and $g^{*}=(\gamma-1) \Lambda^{*} \alpha^{*}$ increases. It is easy to verify that if $m=\bar{M}$ then the corresponding value for $\Lambda^{*}$ is $\Lambda^{*}=\rho+\tau$, and thus $\alpha^{*}=1, \lambda_{l}^{*}=0$ so that we obtain the equilibrium where leaders don't innovate presented in $i i i)$.

The proof of $i i$ ) is straightforward. The right-hand side of (29) is strictly increasing in $m$ so, while keeping $\Lambda^{*}=\bar{\Lambda}$, we can create a one-to-one (strictly increasing) correspondence between $m>\underline{M}$ and $\omega^{*}(a, 1, A)>\xi$. This correspondence defines equilibria with $\lambda_{l}^{*}>0, \Lambda^{*}=\bar{\Lambda}$ and $g^{*}=(\gamma-1) \bar{\Lambda} \alpha^{*}(\bar{\Lambda})$. We have shown above that the equilibrium in $\left.i\right)$ does not exist for $m \geq \bar{M}$. We also know that, by the definition of $\bar{M}$, there is no equilibrium of the type in $i i i)$ for $m>\bar{M}$. Since equilibria must follow under either type $i$ ), ii) or $i i i)$, then for $m>\bar{M}$ the equilibrium of type $i i$ ) is the unique equilibrium.

The existence of an equilibrium of type $i i i)$ for $m \leq \bar{M}$ was part of the definition of $\bar{M}$. The fact that, for a given $m \in\left[\underline{M}, \bar{M}\right.$, the growth for the equilibrium in $i$ ) (call it $g_{i}^{*}$ ) is strictly smaller that the growth in $i i i$ ) (call it $g_{i i i}^{*}$ ) is proven by contradiction. Suppose that $g_{i}^{*} \geq g_{i i i}^{*}$. Then it must be the case that

$$
\begin{gathered}
L_{i}^{*}=1-\frac{g_{i}^{*}}{\gamma-1} \leq 1-\frac{g_{i i i}^{*}}{\gamma-1}=L_{i i i}^{*} \\
r_{i}^{*}=\rho+g_{i}^{*} \geq \rho+g_{i i i}^{*}=r_{i i i}^{*}
\end{gathered}
$$

Since in both cases $\Lambda^{*}<\bar{\Lambda}$, then in both cases $\omega^{*}(a, 1, A)=\xi$, which together with the inequalities above and (??) imply,

$$
\lambda_{f, i}^{*} \leq \lambda_{f, i i i}^{*}
$$

Since leaders innovate at the equilibrium $i$ ), equation (25) holds with equality and then

$$
\gamma \lambda_{i}^{*}=\rho+g_{i i}^{*}
$$

Our discussion leads us to conclude that

$$
\gamma \lambda_{i i i}^{*} \geq \rho+g_{i i i}^{*}
$$

which is a contradiction to the fact that, by construction, leaders strictly prefer not to innovate at equilibria of type $i i i)$ and for $m<\bar{M}$. Finally, in equilibria of type $i$ ) we know that $\Lambda_{i}^{*}<\bar{\Lambda}$ and thus $\alpha^{*}\left(\Lambda_{i}^{*}\right) \Lambda_{i}^{*}>\alpha^{*}(\bar{\Lambda}) \bar{\Lambda}$, proving that the growth rate in $\left.i\right)$ is strictly bigger than the growth rate in $\left.i i\right)$ for $m \in(\underline{M}, \bar{M})$. 


\section{APPENDiX 2. Share $\alpha^{*}$.}

At any instant $t$ call $I_{t} \subseteq[0,1]$ the set of industries in the contestable state. Total productivities $A_{C, t}$ and $A_{N C, t}$ allocated, respectively, to industries in the contestable and non contestable state is

$$
\begin{gathered}
A_{C, t}=\alpha^{*} A_{t} \\
A_{N C, t}=\left(1-\alpha^{*}\right) A_{t}
\end{gathered}
$$

Since arrivals of innovations are i.i.d. across industries we have

$$
\begin{gathered}
A_{C, t+\varepsilon}=\left(1-\lambda_{f}^{*} \varepsilon-\lambda_{l}^{*} \varepsilon\right) A_{C, t}+\gamma \lambda_{f}^{*} A_{C, t} \varepsilon+\tau \varepsilon A_{N C, t}+o(\varepsilon) \\
A_{N C, t}=(1-\tau \varepsilon) A_{N C, t}+\gamma \lambda_{l}^{*} \varepsilon A_{C, t}+o(\varepsilon)
\end{gathered}
$$

where $\lambda_{f}^{*}$ and $\lambda_{l}^{*}$ are the steady state innovation rates of the follower and the leader in an innovative state. Rearranging and taking limits for $\varepsilon \rightarrow 0$ we obtain,

$$
\begin{gathered}
\frac{\dot{A}_{C, t}}{A_{C, t}}=(\gamma-1) \lambda_{f}^{*}-\lambda_{l}^{*}+\tau \frac{1-\alpha^{*}}{\alpha^{*}} \\
\frac{\dot{A}_{N C t}}{A_{N C, t}}=-\tau+\gamma \lambda_{l}^{*} \frac{\alpha^{*}}{1-\alpha^{*}}
\end{gathered}
$$

Since $\alpha^{*}=\left(1+\frac{A_{N C, t}}{A_{C, t}}\right)^{-1}$, a stationary equilibrium with $\alpha^{*}$ constant requires that

$$
\frac{\dot{A}_{C, t}}{A_{C, t}}=\frac{\dot{A}_{N C, t}}{A_{N C, t}}
$$

which implies the following quadratic equation,

$$
(\gamma-1) \Lambda^{*} \alpha^{* 2}-\left(\gamma \lambda_{f}^{*}-\Lambda^{*}-\tau\right) \alpha^{*}-\tau=0
$$

where $\Lambda^{*}=\lambda_{f}^{*}+\lambda_{l}^{*}$ is the equilibrium arrival rate of innovations in an industry at the innovative state. Using, (32) and (33) we have,

$$
A_{t+\varepsilon}=A_{C, t+\varepsilon}+A_{N C, t+\varepsilon}=A_{t}+(\gamma-1) \Lambda^{*} \alpha^{*} A_{t}+o(\varepsilon)
$$

Taking limits, we obtain the balanced growth rate $g^{*}$,

$$
g^{*}=\frac{\dot{A}_{t}}{A_{t}}=(\gamma-1) \Lambda^{*} \alpha^{*}
$$




\section{APPENDIX 3. BOUNDS ON $\gamma$.}

We first derive the bound $\bar{\xi}$ in (7). In any equilibrium we have $r^{*} \geq \rho, \hat{X}^{*} \leq 1, \lambda_{f}^{*} \geq 0, m \leq 2$. The upper value on the value $\gamma \hat{V}_{1}^{l}$ is then

$$
\gamma \hat{V}_{1}^{l} \leq \frac{\hat{w} \gamma}{\rho}
$$

Since $\omega^{*} \geq 1$, a follower cannot innovate if its costs is $\xi(d) \geq \bar{\xi}$

The argument for (13) is developed in a similar way. Since $\tau \geq 0$, the maximum (one-shot deviation) change in value $\gamma \hat{V}_{\tilde{d}+1}^{l}-\hat{V}_{\tilde{d}}^{l}$ for a leader that goes from a non contestable state $\tilde{d} \geq 2$ to a non contestable state $\tilde{d}+1$ is

$$
\gamma \hat{V}_{\tilde{d}+1}^{l}-\hat{V}_{\tilde{d}}^{l}=\frac{(\gamma-1) \hat{w}}{\rho}
$$

If (13) holds then the leader does not want to innovate at states $d_{f} \geq 2$. 